\title{
P-SYSTEMS IN LOCAL NOETHER LATTICES
}

\author{
E. W. JOHNSON
}

Department of Mathematics

University of Iowa

lowa City, Iowa 52242

JOHNNY A. JOHNSON

Department of Mathematics

University of Houston

Houston, Texas 77204

MONTY B. TAYLOR

Department of Mathematics and Computer Science

University of Texas-Pan American

Edinburg, Texas 78539

(Received April 26, 1993 and in revised form September 7, 1993)

\begin{abstract}
In this paper we introduce the concept of a p-system in a local Noether lattice and obtain several characterizations of these elements. We first obtain a topological characterization and then a characterization in terms of the existence of a certain type of decreasing sequence of elements. In addition, p-systems are characterized in quotient lattices and completions.
\end{abstract}

KEY WORDS AND PHRASES. Local, Noether, multiplicative lattice. 1991 AMS SUBJECT CLASSIFICATION CODES. 06F10, 06F05.

In 1962 R. P. Dilworth [7] introduced the concept of a Noether lattice as an abstraction of the lattice of ideals of a Noetherian ring. Many of his ideas have since been extended and have proved to be extremely useful (e.g., [1], [5], [6], [12], and [13]). In this paper we introduce the idea of a p-system in a local Noether lattice and obtain numerous characterizations of these elements. We begin with a topological characterization of p-systems (Theorem 1) and then obtain a characterization of $p$-systems in terms of the existence of a special type of decreasing sequence of elements in the lattice (Theorem 2). Next p-systems are investigated in quotient lattices (Theorem 3) and completions (Theorem 4). When the local Noether lattice under consideration is the lattice of ideals of a local Noetherian ring, a p-system is known as a principal system. The concept of a principal system was introduced by Northcott and Rees [15] and was motivated by Macaulay's well-known theory of inverse systems. As a consequence of our lattice results, we obtain several characterizations of principal systems in rings. Finally we end by giving an example of a local Noether lattice which has a variety of p-systems and which is not the lattice of ideals of any commutative ring.

In general we adopt the terminology of [2], [4], and [7]. Following [7], a local Noether lattice 
is a modular, principally gencrated multiplicative lattice $\mathscr{L}$ which satisfies the ascending chain condition. has a unique maximal clement. and has the property that the greatest element $I$ of $\mathscr{L}$ is a multiplicative identity. Let $\Psi^{\prime}$ he a local Noether lattice with maximal element $M$. An element $\mathrm{Q}$ of $\mathscr{L}^{\mathrm{i}}$ is primary if for all elements $\mathrm{A}$ and $\mathrm{B}$ of $\mathscr{L}, \mathrm{AB} \leq \mathrm{Q}$ implies $\mathrm{A} \leq \mathrm{Q}$ or $\mathrm{B}^{\mathrm{k}} \leq \mathrm{Q}$ for some integer $k$. The radical of an element $A$ of $\mathcal{L}^{\prime}$, denoted by $\operatorname{Rad}(A)$. is defined by

$$
\operatorname{Rad}(A)=V\left\{X+\mathscr{L} \mid X^{\prime} \leq A \text { for some integer } s\right\} .
$$

Furthermore, an element $Q$ of $\Psi^{\prime}$ is said to be $M$-primary if $\operatorname{Rad}(Q)=M .(\operatorname{If} \operatorname{Rad}(Q)=M$, then $Q$ is primary [11. Corollary 2.5. page 191].) For an element $A$ of $\mathscr{L}$, we define $P_{A}$ to be the set $\{\mathrm{Q} \in \mathscr{L} \mid \mathrm{Q}$ is a meet-irreducible M-primary element of $\mathscr{L}$ such that $\mathrm{A} \leq \mathrm{Q}\}$. Also define a metric $\mathrm{d}$ (called the M-adic metric) on $\mathscr{L}$ as follows:

$$
\mathrm{d}(C, D)=0 \text { if }\left(\vee M^{n}=D \vee M^{n} \text { for all nonnegative integers } n\right. \text {; }
$$

otherwise.

$$
\mathrm{d}(C, D)=2^{-\triangleleft(C . D)} \text { where } s(C, D)=\sup \left\{n \mid C \vee M^{n}=D \vee M^{n}\right\} .
$$

This metric gives rise to the $\mathrm{M}$-adic completion of $\mathscr{L}[8]$. Finally an element $\mathrm{A}$ of $\mathscr{L}$ is defined to be a p-system in $\mathscr{L}$ if $A \neq I$ and for every $M$-primary element $Q^{\prime}$ of $\mathscr{L}$ with $A \leq Q^{\prime}$, there exists a meet-irreducible $M$-primary element $\mathrm{Q}$ of $\mathscr{L}$ such that $\mathrm{A} \leq \mathrm{Q} \leq \mathrm{Q}^{\prime}$. Note that all meet-irreducible $\mathrm{M}$-primary elements of $\mathscr{L}$ are $\mathrm{p}$-systems in $\mathscr{L}$.

We begin with the following characterizations of p-systems.

THEOREM 1 . Let $\mathscr{Q}$ be a local Noether lattice with maximal element $M$ and let $A$ be an element of $\mathscr{L}$ different from I. Then the following are equivalent:

(1.1) A is a p-system in $\mathscr{L}$

(1.2) for every positive integer $n$, there exists a meet-irreducible M-primary element $Q$ of $\mathscr{Q}$ such that $\mathrm{A} \leq \mathrm{Q} \leq \mathrm{A} \vee \mathrm{M}^{\mathrm{n}}$

(1.3) $\quad A$ is a closure point of $P_{A}$ in the M-adic topology on $\mathscr{L}$.

PROOF. To show that (1.1) implies (1.2), suppose $A$ is a p-system in $\mathscr{L}$ and $\mathrm{n}$ is a positive integer. Since $M \geq \operatorname{Rad}\left(A \vee M^{n}\right) \geq \operatorname{Rad}\left(M^{n}\right)=M$, it follows that $A \vee M^{n}$ is an $M$-primary element of $\mathscr{L}$. Thus, since $\mathrm{A}$ is a $\mathrm{p}$-system in $\mathscr{L}$, there exists a meet-irreducible M-primary element $\mathrm{Q}$ of $\mathscr{L}$ such $\mathrm{A} \leq \mathrm{Q} \leq \mathrm{A} \vee \mathrm{M}^{\mathrm{n}}$. We now show that (1.2) implies (1.3). Suppose (1.2) holds and $\epsilon>0$. Let $\mathrm{n}$ be a positive integer such that $2^{-\mathrm{n}}<\epsilon$. By (1.2), there exists a meet-irreducible $M$-primary element $Q$ of $\mathscr{L}$ such that $A \leq Q \leq A \vee M^{n}$. It follows that $A \vee M^{n}=Q \vee M^{n}$, and so $Q \in P_{A}$ and $d(A, Q)<2^{-n}<\epsilon$. Hence, $A$ is a closure point of $P_{A}$ in the $M$-adic topology on $\mathscr{L}$. To complete the proof, we show that (1.3) implies (1.1). Suppose A is a closure point of $P_{A}$ in the $\mathrm{M}$-adic topology on $\mathscr{L}$ and that $Q^{\prime}$ is an M-primary element of $\mathscr{L}$ such that $A \leq Q^{\prime}$. Since $\mathrm{Q}^{\prime}$ is $\mathrm{M}$-primary, choose $\mathrm{n}$ to be a positive integer such that $\mathrm{M}^{\mathrm{n}} \leq \mathrm{Q}^{\prime}$. So by (1.3), there exists $Q \in P_{A}$ such that $d(A, Q)<2^{-n}$. Hence, we have that $Q$ is a meet-irreducible M-primary element of $\mathscr{L}$ such that $\mathrm{A} \leq \mathrm{Q}$ and $\mathrm{A} \vee \mathrm{M}^{\mathrm{n}}=\mathrm{Q} \vee \mathrm{M}^{\mathrm{n}}$. Thus, it follows that

$$
\mathrm{A} \leq \mathrm{Q} \leq \mathrm{Q} \vee \mathrm{M}^{\mathrm{n}}=\mathrm{A} \vee \mathrm{M}^{\mathrm{n}} \leq \mathrm{Q}^{\prime} .
$$

Hence, $\mathrm{A}$ is a p-system in $\mathscr{L}$. This completes the proof.

We now characterize principal systems in a local Noether lattice in terms of the existence of a certain type of decreasing requence of special elements in the lattice.

THEOREM 2. Let $\mathscr{L}$ be a local Noether lattice with maximal element $M$ and let $A$ be an element of $\mathscr{L}$ different from $\mathrm{I}$. Then $\mathrm{A}$ is a p-system in $\mathscr{L}$ if and only if there exists a decreasing 
sequence $\left\{Q_{n}\right\}$ of meet-irreducible $M$-primary elements of $\mathscr{L}$ such that

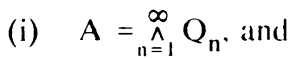

(ii) if $\mathrm{Q}$ is an $\mathrm{M}$-primary element of $\mathscr{L}$ satisfying $\mathrm{A} \leq \mathrm{Q}$, then there is a positive integer $\mathrm{n}$ such that $Q_{n} \leq Q$.

PROOF. Assume there in a decreasing requence $\left\{Q_{n}\right\}$ of elements of $\mathscr{L}$ satisfying conditions (i) and (ii) and suppose $Q^{\prime}$ is in M-primary element of $\mathscr{L}$ such that $A \leq Q^{\prime}$. From condition (ii), we get that there exists a postlue integer n such that $Q_{n} \leq Q^{\prime}$. Also by condition (i), we have that $A \leq Q_{n}$. Therefore, $Q_{n}$ is a meet-irreducible $M$-primary element of $\mathscr{L}$ such that $A \leq Q_{n} \leq Q^{\prime}$. Hence, $A$ is a $p$-system in $\mathscr{L}$. Conversely, assume $A$ is a $p$-system in $\mathscr{L}$. We recursively define a sequence $\left\{Q_{n}\right\}$ of elements of $\mathcal{L}^{\prime}$ as follows: Choose $Q_{1}$ to be $M$. For $n>1$, choose $Q_{n}$ to be a meet-irreducible M-primary element of $\mathscr{L}$ such that

$$
A \leq Q_{n} \leq Q_{n-1} \wedge\left(A \vee M^{n}\right) .
$$

This is possible using (1.2) since $A \leq Q_{n-1} \wedge\left(A \vee M^{n}\right)$ and

$$
\operatorname{Rad}\left(Q_{n-1} \wedge\left(A \vee M^{n}\right)\right)=\operatorname{Rad}\left(Q_{n-1}\right) \wedge \operatorname{Rad}\left(A \vee M^{n}\right)=M
$$

so that $Q_{n-1} \wedge\left(A \vee M^{n}\right)$ is an M-primary element of $\mathscr{L}$. By our construction, $\left\{Q_{n}\right\}$ is a decreasing sequence of meet-irreducible $M$-primary elements of $\mathscr{L}$. Moreover,

$$
A \leq \wedge_{n=1}^{\infty} Q_{n} \leq{ }_{n=1}^{\infty}\left(A \vee M^{n}\right)=A \text {, }
$$

so $\stackrel{n}{n}=1_{n}^{\infty} Q_{n}=A$. Finally, if $Q$ « an $M$-primary element of $\mathscr{L}$ such that $A \leq Q$, then there exists a positive integer $n$ such that $M^{n} \leq Q$, and it follows that $Q_{n} \leq A \vee M^{n} \leq Q$. This completes the proof.

We now recall the definition of quotient lattice given in [7]. Let $\mathscr{L}$ be a Noether lattice and let $\mathrm{D}$ be an element of $\mathscr{L}$. Define $\mathscr{L} / \mathrm{D}$ to be the sublattice $\{\mathrm{X} \in \mathscr{L} \mid \mathrm{D} \leq \mathrm{X}\}$ of $\mathscr{L}$. Then $\mathscr{L} / \mathrm{D}$ is a multiplicative lattice with multiplication $\circ$ defined by

$$
\mathrm{A} \circ \mathrm{B}=\mathrm{AB} \vee \mathrm{D} \text {. }
$$

If $\mathscr{L}$ is local with maximal element $\mathrm{M}$ and $\mathrm{D} \leq \mathrm{M}$, then $\mathscr{L} / \mathrm{D}$ is local with maximal element $\mathrm{M}$; furthermore, for an element $\mathrm{A}$ of $\mathscr{L}$ satisfying $\mathrm{D} \leq \mathrm{A}, \mathrm{A}$ is an $\mathrm{M}$-primary element of $\mathscr{L}$ if and only if $\mathrm{A}$ is an M-primary element of $\mathscr{L} / \mathrm{D}$.

THEOREM 3. Let $\mathscr{L}$ be a local Noether lattice with maximal element $\mathrm{M}$ and let $\mathrm{A}$ be an element of $\mathscr{L}$ different from I. Then the following are equivalent:

(3.1) A is a p-system in $\mathscr{L}$

(3.2) for all elements $\mathrm{B}$ of $\mathscr{L}$ satisfying $\mathrm{B} \leq \mathrm{A}, \mathrm{A}$ is a $\mathrm{p}$-system in $\mathscr{L} / \mathrm{B}$

(3.3) the zero element of $\mathscr{Q} / \mathrm{A}$ is a $\mathrm{p}$-system in $\mathscr{L} / \mathrm{A}$

(3.4) there exists an element $\mathrm{B}$ of $\mathscr{L}$ satisfying $\mathrm{B} \leq \mathrm{A}$ such that $\mathrm{A}$ is a p-system in $\mathscr{L} / \mathrm{B}$.

PROOF. To show that (3.4) implies (3.1), suppose there exists an element $B$ of $\mathscr{L}$ such that $\mathrm{B} \leq \mathrm{A}$ and $\mathrm{A}$ is a p-system in $\mathscr{L} / \mathrm{B}$. In addition, suppose that $\mathrm{n}$ is a positive integer. Then using (1.2), there exists a meet-irreducible M-primary element $Q$ of $\mathscr{L} / B$ such that $A \leq Q \leq A \vee M^{n}$. Hence, we get that $Q$ is a meet-irreducible M-primary element of $\mathscr{L}$ such that $A \leq Q \leq A \vee M^{\mathbf{n}}$. Therefore, $\mathrm{A}$ is a p-system in $\mathscr{\mathcal { W }}$. The proofs that (3.1) implies (3.2), that (3.2) implies (3.3), and that (3.3) implies (3.4) are straightforward and we omit the details. This completes the proof.

For a local Noether lattice $\mathscr{L}$ with maximal element $M$, we now investigate $p$-systems in the completion of $\mathscr{Q}$ with respect to the M-adic metric described earlier. Following [8], let $\mathscr{Q}^{*}$ denote the set of all formal sums $\sum_{i=1}^{\infty} A_{i}$ of elements of $\mathscr{L}$ such that 


$$
A_{i}=A_{i+1} \vee M^{i}
$$

for all positive integers $i$. On $\mathscr{L}^{*}$. define

$$
\sum_{i=1}^{\infty} A_{1} \leq \sum_{i=1}^{\infty} B_{i} \text { if and only if } A_{i} \leq B_{i} \text { for all } i
$$

and

$$
\left(\sum_{i=1}^{\infty} A_{i}\right)\left(\sum_{i=1}^{\infty} B_{i}\right)=\sum_{i=1}^{\infty}\left(A_{i} B_{i} \vee M^{i}\right)
$$

For an element $A$ of $\mathscr{L}$, let $A^{\prime}$ denote the element $\sum_{i=1}^{\infty}\left(A \vee M^{i}\right)$ of $\mathscr{L}^{*}$. Then $\mathscr{L}^{*}$ is a local Noether lattice with maximal element $M^{*}=\sum_{i=1}^{\infty} M$. It can be seen that $\mathscr{L}^{*}$ is a collection of representatives of equivalence classes of Cauchy sequences of $\mathscr{L}$ with the $\mathrm{M}$-adic metric and in fact is the completion of $\mathscr{L}$ with this metric. If $\sum_{i=1}^{\infty} B_{i}$ is an element of $\mathscr{L}^{*}$, then $C\left(\sum_{i=1}^{\infty} B_{i}\right)$ is the element $\hat{i=1}_{i=1}^{\infty} B_{i}$ of $\mathscr{L}$. For each positive integer $\mathrm{I}$, the map $A \rightarrow A^{*}$ from $\mathscr{L} / \mathbf{M}^{i}$ to $\mathscr{Q}^{*} /\left(\mathbf{M}^{*}\right)^{i}$ and the map $B \rightarrow C(B)$ from $\mathscr{Q}^{*} /\left(\mathrm{M}^{*}\right)^{\mathrm{i}}$ to $\mathscr{Q} / \mathrm{M}^{\mathrm{i}}$ are multiplicative lattice isomorphisms. Additional properties can be found in $[8]-[10]$.

THEOREM 4. Let $\mathscr{L}$ be a local Noether lattice with maximal element $M$ and let $A$ be an element of $\mathscr{L}$ different from $\mathrm{I}$. Then $\mathrm{A}$ is a p-system in $\mathscr{L}$ if and only if $\mathrm{A}^{*}$ is a p-system in $\mathscr{L}^{*}$.

PROOF. Suppose $A$ is a principal system in $\mathscr{L}$ and $n$ is a positive integer. Then by (1.2) there exists a meet-irreducible $M$-primary element $Q$ of $\mathscr{L}$ such that $A \leq Q \leq A \vee M^{n}$. Thus, it follows that $A^{*} \leq Q^{*} \leq A^{*} \vee\left(M^{*}\right)^{n}$. Since $M=\operatorname{Rad}(Q)$, pick a positive integer $k$ such that $\mathbf{M}^{\mathbf{k}} \leq \mathrm{Q}$. Since $\mathscr{L} / \mathrm{M}^{\mathbf{k}}$ is isomorphic to $\mathscr{L}^{*} /\left(\mathrm{M}^{*}\right)^{\mathbf{k}}$, it follows that $\mathrm{Q}^{*}$ is a meet-irreducible $\mathbf{M}^{*}$-primary element of $\mathscr{L}^{*}$. Thus, by (1.2), $\mathrm{A}^{*}$ is an p-system in $\mathscr{L}^{*}$.

Conversely, suppose $A^{*}$ is a p-system in $\mathscr{L}^{*}$ and $n$ is a positive integer. Then by (1.2) there exists a meet-irreducible $M^{*}$-primary element $Q^{\prime}$ of $\mathscr{L}^{*}$ such that $A^{*} \leq Q^{\prime} \leq A^{*} \vee\left(M^{*}\right)^{n}$. Thus, it follows that

$$
\mathrm{A}=\mathrm{C}\left(\mathrm{A}^{*}\right) \leq \mathrm{C}\left(\mathrm{Q}^{\prime}\right) \leq \mathrm{C}\left(\mathrm{A}^{*} \vee\left(\mathrm{M}^{*}\right)^{\mathrm{n}}\right)=\mathrm{A} \vee \mathrm{M}^{\mathrm{n}} .
$$

Since $M^{*}=\operatorname{Rad}\left(Q^{\prime}\right)$, pick a positive integer $r$ such that $\left(M^{*}\right)^{r} \leq Q^{\prime}$. Therefore, we have that $\mathbf{M}^{\mathbf{r}}=\mathrm{C}\left(\left(\mathbf{M}^{*}\right)^{\mathrm{r}}\right) \leq \mathrm{C}\left(\mathrm{Q}^{\prime}\right)$ and since $\mathscr{L}^{*} /\left(\mathrm{M}^{*}\right)^{\mathrm{r}}$ is isomorphic to $\mathscr{L} / \mathbf{M}^{\mathrm{r}}$, we get that $\mathrm{C}\left(\mathrm{Q}^{\prime}\right)$ is a meet-irreducible M-primary element of $\mathscr{L}$. Hence, by (1.2), $\mathrm{A}$ is a principal system in $\mathscr{L}$. This completes the proof.

We now summarize the results of the previous theorems.

THEOREM 5. Let $\mathscr{L}$ be a local Noether lattice with maximal element $M$ and let $A$ be an element of $\mathscr{L}$ different from $\mathrm{I}$. Then the following are equivalent:

(5.1) A is a p-system in $\mathscr{L}$

(5.2) for every positive integer $n$, there exists a meet-irreducible $M$-primary element $Q$ of $\mathscr{L}$ such that $\mathrm{A} \leq \mathrm{Q} \leq \mathrm{A} \vee \mathrm{M}^{\mathrm{n}}$

(5.3) A is a closure point of $P_{A}$ in the M-adic topology on $\mathscr{L}$

(5.4) there exists a decreasing sequence $\left\{Q_{n}\right\}$ of meet-irreducible $M$-primary elements of $\mathscr{L}$ such that

(i) $\quad \mathrm{A}=\stackrel{\wedge}{n=1}_{n} \mathrm{Q}_{\mathrm{n}}$, and

(ii) if $\mathrm{Q}$ is an $\mathrm{M}$-primary element of $\mathscr{L}$ satisfying $\mathrm{A} \leq \mathrm{Q}$, then there is a positive integer $n$ such that $Q_{n} \leq Q$

(5.5) for all elements $\mathrm{B}$ of $\mathscr{L}$ satisfying $\mathrm{B} \leq \mathrm{A}, \mathrm{A}$ is a p-system in $\mathscr{L} / \mathrm{B}$

(5.6) the zero element of $\mathscr{L} / \mathrm{A}$ is a p-system in $\mathscr{L} / \mathrm{A}$

(5.7) there exists an element $\mathrm{B}$ of $\mathscr{L}$ satisfying $\mathrm{B} \leq \mathrm{A}$ such that $\mathrm{A}$ is a p-system in $\mathscr{L} / \mathrm{B}$

(5.8) $\mathrm{A}^{*}$ is a p-system in $\mathscr{Q}^{*}$. 
We now turn our attentuon to rings where in general we adopt the terminology of [14]. Let $\mathbf{R}$ be a local Noetherian ring with maximal ideal $M$. Then $\mathscr{L}(\mathbf{R})$, the lattice of ideals of $\mathbf{R}$, is a local Noether lattice. We say that an ideal $A$ of $R$ is irreducible if $A$ is a meet-irreducible element of $\mathscr{Q}(R)$. In addition, an ideal $A$ of $R$ is a principal system in $R$ if and only if $A$ is a $p$-system in $\mathscr{L}(R)$. Let $R^{*}$ denote the ring $M$-adic completion of $R$ and let $A R^{*}$ denote the (ring) extension of an ideal $A$ of $R$ to $R^{*}$. Thus, we obtain the following result.

THEOREM 6 . Let $R$ be a local Noetherian ring with maximal ideal $M$ and let $A$ be $a$ proper ideal of $R$. Then the following are equivalent:

(6.1) A is a principal system in $R$

(6.2) for every positive integer $n$, there exists an irreducible $M$-primary ideal $Q$ of $R$ such that $\mathrm{A} \subseteq \mathrm{Q} \subseteq \mathrm{A}+\mathrm{M}^{\mathrm{n}}$

(6.3) $A$ is a closure point of $P_{A}$ in the $M$-adic topology on $\mathscr{L}(R)$

(6.4) there exists a decreasing sequence $\left\{Q_{n}\right\}$ of irreducible $M$-primary ideals of $R$ such that

(i) $\quad A=\bigcap_{n=1}^{\infty} Q_{n}$, and

(ii) if $Q$ is an M-primary ideal of $R$ satisfying $A \subseteq Q$, then there is a positive integer $n$ such that $\mathrm{Q}_{\mathrm{n}} \subseteq \mathrm{Q}$

(6.5) for all ideals $B$ of $R$ satisfying $B \subseteq A, A$ is a principal system in $R / B$

(6.6) the zero ideal of $R / A$ is a principal system in $R / A$

(6.7) there exists an ideal $B$ of $R$ satisfying $B \subseteq A$ such that $A$ is a principal system in $R / B$

(6.8) $\mathrm{AR}^{*}$ is a principal system in $\mathrm{R}^{*}$.

PROOF. The equivalences of (6.1)-(6.7) follows from (5.1)-(5.7) and the fact that $Q$ is an irreducible $M$-primary ideal of $R$ if and only if $Q$ is a meet-irreducible $M$-primary element of $\mathscr{L}(R)$. To show that (6.1) and (6.8) are equivalent, we have the following chain of equivalences:

$A$ is a principal system in $R$

$\Leftrightarrow \quad A$ is a p-system in $\mathscr{L}(R)$

$\Leftrightarrow \quad A^{*}$ is a p-system in $\mathscr{L}(R)^{*}$

$\Leftrightarrow \quad \mathbf{A R}^{*}$ is a p-system in $\mathscr{L}\left(\mathbf{R}^{*}\right)$

$\Leftrightarrow \quad \mathrm{AR}^{*}$ is a principal system in $\mathrm{R}^{*}$.

The second equivalence follows from the equivalence of (5.1) and (5.8), whereas the third equivalence follows from the fact that there exists an isomorphism $\varphi: \mathscr{L}(\mathbf{R})^{*} \rightarrow \mathscr{L}\left(\mathbf{R}^{*}\right)$ with the property that $\varphi\left(A^{*}\right)=A^{*}$ (see [10, Theorem 3, p. 158] and its proof). This completes the proof.

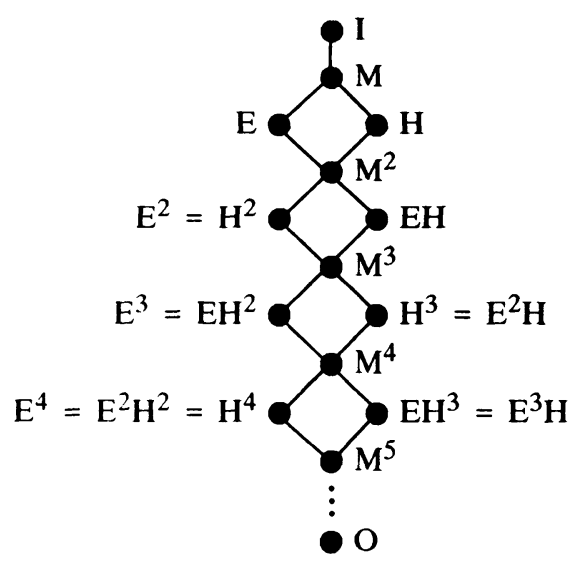


We conclude this paper by giving an example of a local Noether lattice which is not the lattice of Ideals of any commutative ring and which has a variety of principal systems. Let $\mathscr{L}$ be the sublattice of $R L_{2}[3]$ pictured above. It is easily seen that all elements of $\mathscr{L}$ except for $I$ and $O$ are M-primary elements of $\mathcal{L}$. In addition. the maximal element $M$ as well as all elements of the form $E^{m}$ (where $\left.m>0\right)$ or $E^{n} H$ (where $n \geq 0$ ) are p-systems since these elements are also meet-irreducible. However, the least element $O$ is another p-system in $\mathscr{L}$ since the sequence $\left\{E^{n}\right\}$ satisfies the conditions of Theorem 2 and $O$ is the meet of the elements of this sequence. Finally, since $\mathscr{L}$ is distributive and is not a chain, it follows [3, Theorem 3, p. 222] that $\mathscr{L}$ is not the lattice of ideals of any commutative ring.

\section{REFERENCES}

1. ANDERSON, D. D. Abstract commutative ideal theory without chain conditions, Algebra Universalis $\underline{6}$ (1976), 131-145.

2. BIRKHOFF, G. Lattice Theory, 3rd ed., American Mathematical Society, Providence, 1967.

3. BOGART. K. P. Distributive local Noether lattices, Mich. J. Math 16 (1969), 215-223.

4. CRAWLEY, P. and R. P. DILWORTH Algebraic Theory of Lattices, Prentice-Hall, Inc., Englewood Cliffs, New Jersey, 1973.

5. DETLEFSEN, M. E. Regularity in terms of reductions in local Noether lattices, Proc. Am. Math. SOC. $\underline{43}$ (1974), 1-7.

6. DETLEFSEN, M. E. Symmetric sublattices of a Noether lattice, Pacific J. Math. $\underline{77}$ (1978), 365-379.

7. DILWORTH, R. P. Abstract commutative ideal theory, Pacific J. Math. 12 (1962), 481-498.

8. JOHNSON, E. W. and J. A. JOHNSON M-primary elements of a local Noether lattice, Canad. J. Math 22 (1970), 327-331.

9. JOHNSON, J. A. A note on regular local Noether lattices, Glasgow Math. J. 15 (1974), 159-161.

10. JOHNSON, J. A. A note on regular local Noether lattices II, Glasgow Math. $\underline{\mathbf{J}} \underline{18}$ (1977), 157-159.

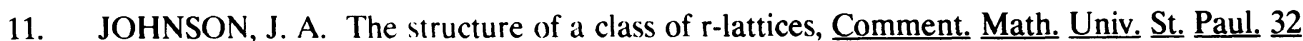
(1983), 189-194.

12. MCCARTHY, P. J. Arithmetic rings and multiplicative lattices, $\underline{\text { Ann. }} \underline{\text { Mat. Pura }} \underline{\text { Appl. }} \underline{82}$ (1969), 267-274.

13. MCCARTHY, P. J. Principal elements of lattices of ideals, Proc. Am. Math. Soc. $\underline{30}$ (1971), 43-45.

14. NAGATA, M. Local Rings, Interscience, New York, 1962.

15. NORTHCOTT, D. G. and D. REES Principal systems, Quart. J. Math. Oxford $\underline{8}$ (1957), 119-127. 


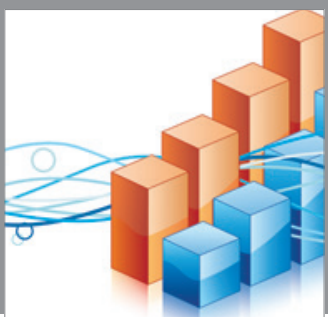

Advances in

Operations Research

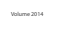

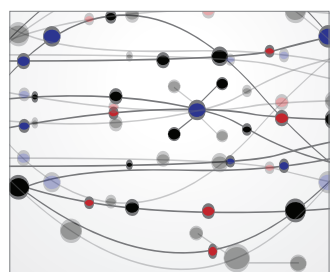

\section{The Scientific} World Journal
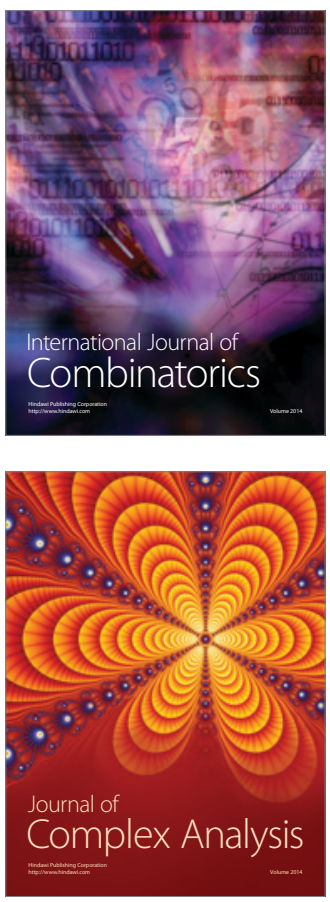

International Journal of

Mathematics and

Mathematical

Sciences
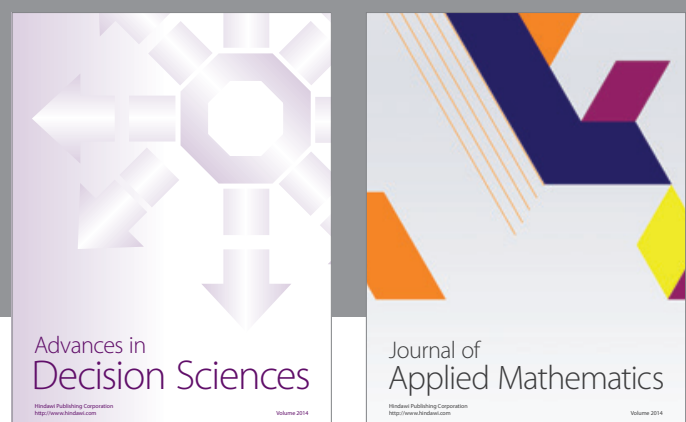

Journal of

Applied Mathematics
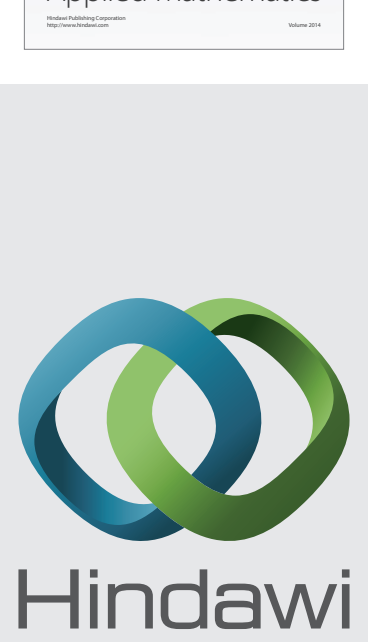

Submit your manuscripts at http://www.hindawi.com
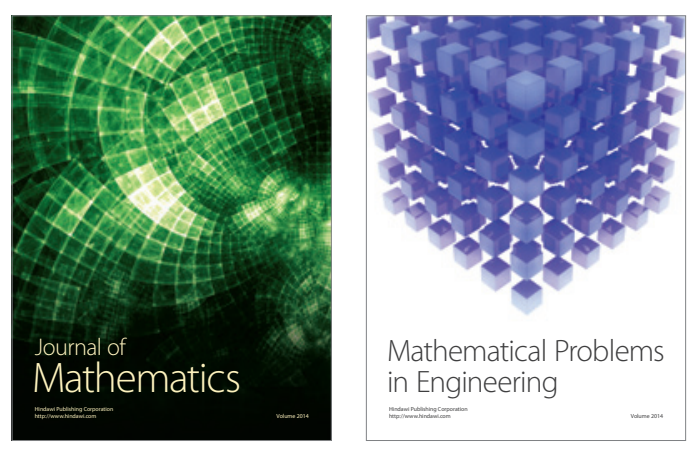

Mathematical Problems in Engineering
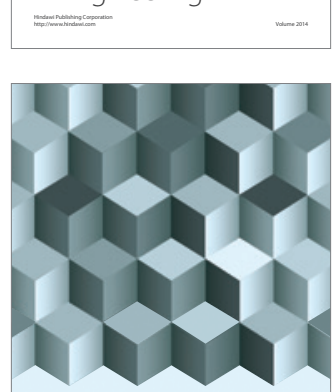

Journal of

Function Spaces
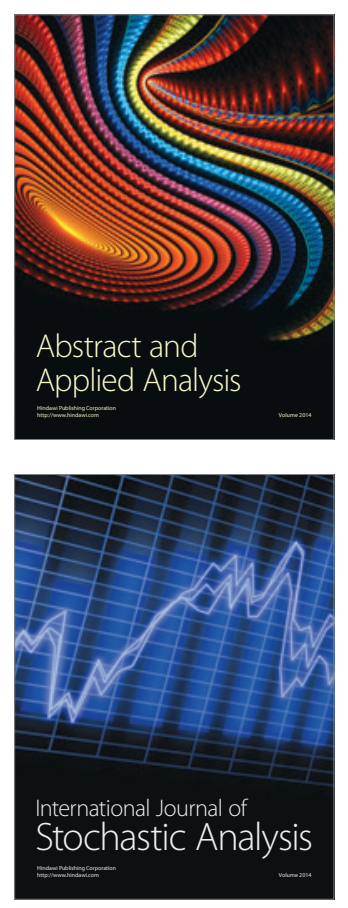

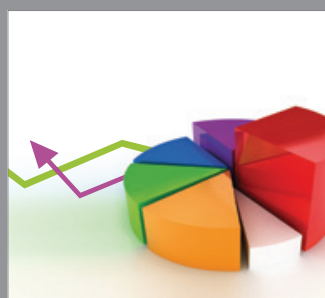

ournal of

Probability and Statistics

Promensencen
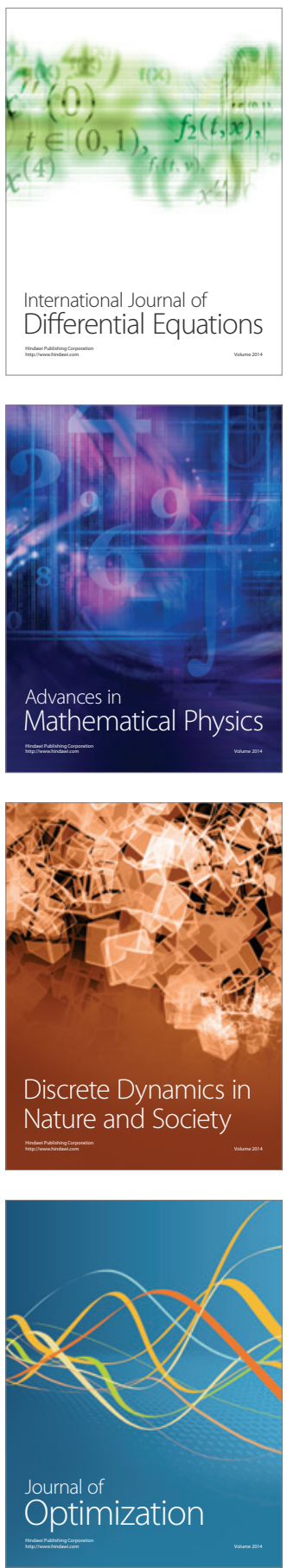